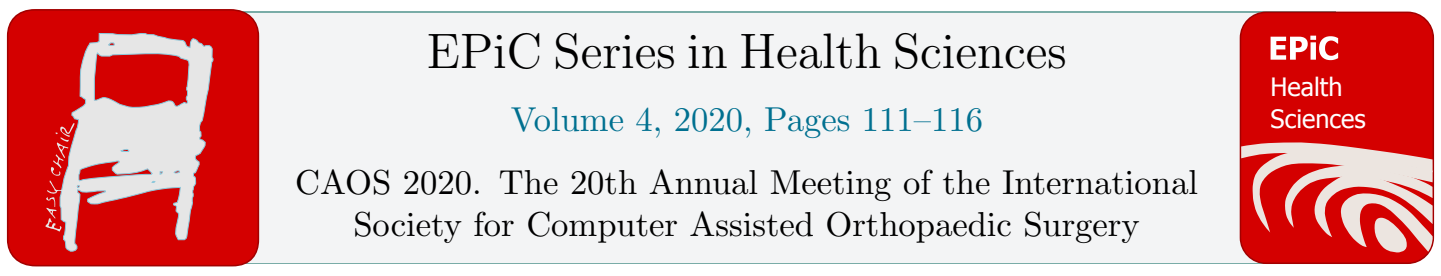

\title{
The Effects of Robotic Assisted TKA: A Retrospective Evaluation of Key Metrics
}

\author{
Jeffrey Hodrick ${ }^{1}$, Thomas Schlierf ${ }^{1}$, Jeffrey Pearson ${ }^{1}$, and Mary Stumb ${ }^{1}$ \\ ${ }^{1}$ Southern Joint Replacement Institute, Nashville, TN \\ jhodrick@gmail.com
}

\begin{abstract}
As technology drives improvement in healthcare, the utilization trends for robotics in arthroplasty has continued to increase over the last decade. With the growth of robotics, we must determine if the proposed benefits are worth the increased cost. The purpose of this study was to evaluate inpatient post-operative and post-discharge outcomes of robotic-assisted surgery vs. conventional manual instrumentation, specifically time to discharge, discharge status and opioid consumption postoperatively. Knee Injury and osteoarthritis outcome score (KOOS) was calculated for each group as well.

After IRB approval, a retrospective chart review of 100 robotic assisted primary total knee arthroplasty (TKA) and 100 matched controls undergoing conventional TKA was performed. Baseline demographics were recorded as well as post-operative outcomes including length of stay, opioid consumption, discharge status and duration of opioid use. All patients underwent primary TKA from 2016-2018 with minimum 6month follow-up by a single fellowship trained arthroplasty surgeon at a high-volume joint center. Exclusion criteria included $<6$ month $\mathrm{f} / \mathrm{u}$, incomplete chart information, inflammatory arthritis, BMI $>40$.

Patients had similar pre-operative demographics including age, BMI, gender, opioid use and baseline depression rates. The robotic assisted TKA group had statistically significant decreased LOS (1.58 vs. $2.18 \mathrm{p}<0.001)$ and morphine equivalents during their hospital stay (73.52 vs. $102.50 \mathrm{p}<0.02)$. The robotic group had fewer patients at six weeks requiring opioids (37 vs. $61 \mathrm{p}=0.001$ ). Six month postoperative KOOS was 81.73 in the control group and 78.22 in the robotic group, $(\mathrm{P}>0.05)$.

Robotic-assisted TKA was associated with statistically significant decreased hospital LOS, morphine equivalents, and opioid usage at the 6-week follow up appointment. KOOS for the groups in our study were not statistically different. Although KOOS in the control group trended to be higher, our average KOOS for both cohorts was higher than the national average of 76.8 .
\end{abstract}




\section{Introduction}

Technology continues to drive improvements in modern healthcare. Utilization trends for technology assistance in total joint arthroplasty continue to increase over the past decade (Boylan M, 2018:33) (Lehil, 2014: 29(10)) (Hsiue, 2020). The use of robotic-assisted surgery started in the 1980's and has grown exponentially in joint arthroplasty (Lang JE, 2011) (Bargar, 2007) (Gourin G, 2007). Despite the growth of robotics in arthroplasty, there is concern that the associated costs may outweigh the proposed benefits that accompany this technology. Robotic assisted total knee arthroplasty (TKA) has been used to improve clinical outcomes, implant survivorship, component alignment/positioning, bone preparation, soft tissue balance and protection, and to decrease the $15-20 \%$ of patients who reported being dissatisfied with their joint replacement (Noble PC, 2006). It has been extensively reported in literature that robotic-assisted TKA (RA-TKA) yields more accurate and precise bone cuts producing consistent and accurate post-operative mechanical alignment compared to manual instrumentation (Bellemans J, 2007) (Song EK, 2013) (Borner M, 2004) (Mai S, 2004) (Decking J, 2004) (Hampp EL, 2019).

As the number of arthroplasty cases continues to increase there is an increased awareness of opioid use and post-operative pain control (Bedard, 2017) (Politzer, 2018). Centers have become increasingly attentive to the dangers of opioids and the need to limit their use post operatively as they can result in increased complications (Sing, 2016). As a result, the field has changed over the last decade to use a variety of modalities to assist in post-operative pain control (Lamplot, 2014) (Gwam, 2017) (Mullaji, 2010).

With the growing literature supporting the use of robotic assisted arthroplasty, there is still much to learn on the outcomes of this technology. Some reviews have shown decreased pain scores following robotic assisted arthroplasty, but relatively few have reported on actual opioid consumption itself (Marchand, 2019) (Kayani, 2019). The purpose of this study was to evaluate inpatient postoperative and post-discharge outcomes of robotic-assisted surgery vs. conventional manual instrumentation, specifically time to discharge, discharge status and opioid consumption postoperatively.

\section{Materials and Methods}

After institutional review board approval, a retrospective study was performed evaluating outcomes of patients undergoing robotic-assisted total knee arthroplasty at a single high-volume joint institution. One hundred patients were identified that underwent a robotic-assisted $(\mathrm{R})$ primary total knee arthroplasty following a transition period to all robotic-assisted. A second matched group was identified, control group (C), consisting of 100 patients who underwent primary total knee arthroplasty with manual instrumentation immediately prior to transitioning to all robotic-assisted. A total of 200 patients were reviewed, all having received a primary TKA between 2016 and 2018 with the minimum of a 6-month follow-up. Patients were selected consecutively excluding those that did not meet the study inclusion and exclusion criteria. All surgeries were performed by 1 fellowship trained arthroplasty surgeon (author J.H.). Patient group R was compared to matched control group C. A single implant design (Triathlon; Stryker, Mahwah, NJ) was used for all patients in this study. Both groups were managed with the same post-operative protocols. All patients at our institution receive spinal anesthesia with adductor canal block, periarticular injection and a limited opioid post-operative pain protocol. Post-operative pain scores were assessed and recorded per institution's post-operative protocols.

Study inclusion criteria included 18-79 years of age, primary TKA performed between 2016-2018 with at least 6-months of clinical follow-up to measure outcomes (KOOS Jr.), and a Triathlon implant 
design. Exclusionary criteria included age $<17$ and $>79$, BMI $>40$, inflammatory arthritis, follow-up $<$ 6 months, patients with an active infection or suspected latent infection in or about the knee joint, simultaneous bilateral TKAs, staged bilateral TKAs performed less than 6 months apart, workers' compensation cases, or patients with inadequate bone stock to support fixation of the prosthesis.

Data analyzed included: length of stay (LOS), ability to walk $>50$ feet during inpatient physical therapy, surgical time, pain scores, morphine equivalents, opioid status at 6 weeks, inpatient and postdischarge complications, and demographics. Pre-op and 6-month Post-op Knee Injury and osteoarthritis outcome score, junior (KOOS Jr.) were calculated for each group. Independent samples t-test and Chi square analysis were used to determine statistical significance. A p-value of $<0.05$ was determined to be statistically significant for this study.

\section{Results}

Demographic data was assessed for variance with no significant differences between the roboticassisted and conventional groups for the pre-operative variables including age, body mass index (BMI), and gender (Table 1). Insurance status was similar for each group as well with 52 patients having Medicare in the control group and 51 in the robotic-assisted group. The length of stay (LOS) and morphine equivalents were significantly less in group R. LOS in group R was 1.58 days $(\mathrm{SD}=0.58)$ versus the control at 2.18 days $(\mathrm{SD}=0.44)(\mathrm{p}=<0.001)$, nearly half a day longer. Morphine equivalents for group $\mathrm{C}$ were nearly twice as great $(\mathrm{M}=102.50, \mathrm{SD}=95.60)$ compared to group $\mathrm{R}$ $(\mathrm{M}=73.51, \mathrm{SD}=69.78)(\mathrm{p}=0.017)$. Post-operative opioid use at 6 weeks was significantly greater for group $\mathrm{C}$ with 61 patients still taking opioids compared with 37 patients in group $\mathrm{R}(\mathrm{p}=0.001)$. The surgical $(\mathrm{p}=<0.013)$ and tourniquet $(\mathrm{p}=<0.019)$ times were statistically significantly greater for group $\mathrm{R}$ (155.17 and 46.68 mins) compared to group C (147.86 and 42.74 mins) (Table 1). No other statistically significant differences were found between the two groups regarding 18-hour postoperative pain, patients who walked over 50 feet during inpatient physical therapy, or the number of inpatient and post-discharge complications. There was no statistically significant difference for the KOOS Jr. between our groups (Table 1). Although the 6-month post-op KOOS Jr. for group C (78.22) was higher than group R (81.73), both were higher compared to the national average (76.8) (Table 2).

\section{Discussion}

Post-operative outcome measures are multifactorial with each potentially having an individual effect. Identifying such factors and determining if modifications can be made to improve patient care may not only serve to benefit the patient but also can have a ripple effect in our healthcare system. This study showed that the robotic-assisted group had statistically significant decreases in LOS, morphine equivalents, and 6-week opioid use. Total surgical and tourniquet time, however, were longer when compared to the manual instrumented group. Haddad et al had similar results showing less post-operative pain, less time to discharge, and decreased analgesia requirements when comparing robotic-arm assisted TKA to conventional instrumentation TKA (Kayani B, 2018). Another study by Marchand et al reported significantly lower mean pain scores comparing roboticarm assisted-TKA to manual instrumentation TKA (Marchand RC, 2017) (Bhimani, 2020). These findings may be due to the differences in surgical technique provided with robotic-assisted surgery such as limiting soft tissue releases, intramedullary violation, and reduced bone and periarticular soft tissue injury. Several studies have shown increased pain and delayed post-operative rehabilitation can result from even limited soft tissue releases which may promote changes in local and systemic 
inflammatory responses (Laskin RS, 2004) (Niki Y, 2009) (Bradley B, 2017) (Cody JP, 2017). Decreasing opioid requirements can reduce cost, as well as minimize associated post-operative complications, to make a positive impact in the opioid crisis seen in healthcare today (Bedard NA, 2018) (Wilson JM, 2019).

There are several limitations to this paper. Being a non-randomized retrospective study, it is subject to the biases inherent in its design. Selection bias is a consideration when comparing outcomes as certain patients were selected to undergo robotic assisted arthroplasty. This review also had fairly short follow up of 6 months. Patients were also not blinded in the surgery they received, i.e. roboticassisted or conventional methods. The patients therefore could have an inherent placebo bias and thus decreased pain perception because of this. Despite these limitations, this is a retrospective single surgeon study using the same robotic platform; implant design, surgical approach, and post-operative pain and rehabilitation protocols. Robotic-assisted TKA was associated with statistically significant decreased hospital length of stay, morphine equivalents, and opioid usage at the 6-week follow up appointment. When compared to the national average, the KOOS Jr. for both of our cohorts were higher than the national average.

Further long-term investigation is needed to assess the financial and functional implications of robotic-assisted surgery. This study provides early clinical support that robotic-assisted surgery may contribute to an overall opioid reduction strategy.

\section{Appendix}

Table 1. Mean, Standard Deviation, and P Values of Data Metrics for Group R and Group C

\begin{tabular}{llllll}
\hline & $\begin{array}{l}\text { Robotic } \\
(\mathrm{R})\end{array}$ & $\begin{array}{l}\text { Standard } \\
\text { Deviation }\end{array}$ & $\begin{array}{l}\text { Control } \\
(\mathrm{C})\end{array}$ & $\begin{array}{l}\text { Standard } \\
\text { Deviation }\end{array}$ & P Value \\
\hline Demographics & & & & & \\
$\quad$ Age & 65.59 & 8.57 & 66.01 & 8.15 & $>0.05$ \\
$\quad$ Gender (M:F) & $46: 54$ & & $44: 57$ & & $>0.05$ \\
$\quad$ BMI & 31.01 & 4.53 & 30.15 & 4.37 & $>0.05$ \\
Length of Stay (days) & 1.58 & 0.58 & 2.18 & 0.44 & $<0.001$ \\
Morphine Equivalence & 73.52 & 69.78 & 102.50 & 96.51 & 0.017 \\
Opioid Use at 6 Weeks (Y:N) & $37: 63$ & & $61: 39$ & & 0.001 \\
Surgical Time (min) & 155.17 & 18.98 & 147.86 & 22.00 & 0.013 \\
Tourniquet Time (min) & 46.68 & 9.80 & 42.74 & 13.00 & 0.019 \\
Pre-Op KOOSjr & 46.72 & 12.63 & 47.62 & 13.43 & $>0.05^{*}$ \\
6 Month Post-Op KOOSjr & 78.22 & 20.44 & 81.73 & 18.90 & $0.039^{*}$
\end{tabular}

Table 2. National KOOSjr Scores

\begin{tabular}{ll}
\hline & Mean Score \\
\hline National Pre-Op KOOSjr & 46.5 \\
National 1 Year Post-Op KOOSjr & 76.8
\end{tabular}




\section{References}

Bargar, W. (2007). Clin Orthop Relat Res. Robots in orthopedic surgery, 463:31-6.

Bedard NA, D. D. (2018). Preoperative Opioid Use and Its Association With Early Revision of Total Knee Arthroplasty. The Journal of Arthroplasty, 33(11):3520-3523. doi:10.1016/j.arth.2018.06.005.

Bedard, N. A. (2017). Opioid use after total knee arthroplasty: trends and risk factors for prolonged use. The Journal of arthroplasty, 32(8), 2390-2394.

Bellemans J, V. H. (2007). Robot-assisted total knee arthroplasty. Clin Orthop Relat Res, 464:111.

Bhimani, S. J. (2020). Robotic-assisted total knee arthroplasty demonstrates decreased postoperative pain and opioid usage compared to conventional total knee arthroplasty. Bone and Joint, 1:2, 8-12.

Borner M, W. U. (2004). Clinical experiences with Robodoc and the Duracon Total Knee. In: Stiehl J, Konermann W, Haaker R, editors. Navigation and robotics in total joint and spine surgery. Berlin, Germany: Springer-Verlag, p. 362.

Boylan M, S. K.-1. (2018:33). Technology-Assisted Hip and Knee Arthroplasties: An Analysis of Utilization Trends. Journal of Arthroplasty, 1019-1023.

Bradley B, M. S. (2017). Discharge on the day of surgery following unicompartmental knee arthroplasty within the United Kingdom NHS. Bone Joint J, 99-B: 788-92.

Cody JP, P. K. (2017). Is outpatient unicompartmental knee arthroplasty safe to perform at an ambulatory surgery center? A comparative study of early post-operative complications. The Journal of Arthroplasty, 33:10-6.

Decking J, T. C. (2004). Robotic total knee arthroplasty: the accuracy of CT-based component placement. Acta Orthop Scand, 75:573.

Gourin G, T. J. (2007). History of robotic surgery. In: Faust RA, editor. Robotics in surgery: history, current and future applications. New York, NY: Nova Science Publishers, Inc, 3-12.

Gwam, C. U. (2017). Does addition of multimodal periarticular analgesia to adductor canal block improve lengths of stay, pain, discharge status, and opioid use after total knee arthroplasty? The Journal of Arthroplasty, 32(5), 1470-1473.

Hampp EL, C. M.-S. (2019). Robotic-arm assisted total knee arthroplasty demonstrated greater accuracy and precision to plan compared with manual techniques. J Knee Surg, 32(3):23925. doi: 10.1055/s-0038- 1641729. Epub 2018 May 1. .

Hsiue, P. P. (2020). Trends and patient factors associated with technology-assisted total hip arthroplasty in the United States from 2005 to 2014. Arthroplasty Today.

Kayani B, K. S. (2018). Iatrogenic bone and soft tissue trauma in robotic-arm assisted total knee arthroplasty: A prospective cohort study and validation of a new classification system. The Journal of Arthroplasty, 33(8):2496-2501.

Kayani, B. K. (2019). An assessment of early functional rehabilitation and hospital discharge in conventional versus robotic-arm assisted unicompartmental knee arthroplasty: a prospective cohort study. Bone Joint J, 101(1), 24-33.

Lamplot, J. D. (2014). Multimodal pain management in total knee arthroplasty: a prospective randomized controlled trial. The Journal of Arthroplasty, 29(2), 329-334.

Lang JE, M. S. (2011). Robotic systems in orthopedic surgery. Bone Joint, 93-B:292-9 .

Laskin RS, B. B. (2004). Minimally invasive total knee replacement through a mini-midvastus incision: an outcome study. Clin Orthop Relat Res, 74e81.

Lehil, M. S. (2014: 29(10)). Trends in total hip arthroplasty implant utilization in the United States. The Journal of Arthroplasty, 1915-1918.

Mai S, L. C. (2004). Clinical results with the robot assisted Caspar System and the Search-Evolution Prosthesis. In: Stiehl J, Konermann W, Haaker R, editors. Navigation and robotics in total joint and spine surgery. Berlin, Germany: Springer Verlag, p. 355. 
Marchand RC, S. N. (2017). Patient satisfaction outcomes after robotic arm-assisted total knee arthroplasty: a short-term evaluation. J Knee Surg, 0:849-53.

Marchand, R. C. (2019). One-year patient outcomes for robotic-arm-assisted versus manual total knee arthroplasty. The journal of knee surgery, 32(11), 1063-1068.

Mullaji, A. K. (2010). Efficacy of periarticular injection of bupivacaine, fentanyl, and methylprednisolone in total knee arthroplasty: a prospective, randomized trial. The Journal of Arthroplasty, 25(6), 851-857.

Niki Y, M. T. (2009). Is minimally invasive surgery in total knee arthroplasty really minimally invasive surgery? The Journal of Arthroplasty, 24:499-504.

Noble PC, C. M. (2006). The John Insall Award: patient expectations affect satisfaction with total knee arthroplasty. Clin Orthop Relat Res, 452:35-43.

Politzer, C. S. (2018). Trends in opioid utilization before and after total knee arthroplasty. The Journal of Arthroplasty, 33(7), S147-S153.

Sing, D. C. (2016). Long-acting opioid use independently predicts perioperative complication in total joint arthroplasty. The Journal of Arthroplasty, 31(9), 170-174.

Song EK, S. J. (2013). Robotic-assisted TKA reduces postoperative alignment outliers and improves gap balance compared to conventional TKA. Clin Orthop Relat Res, 471(1):118.

Wilson JM, F. K. (2019). The Impact of Preoperative Opioid Use Disorder on Complications and Costs following Primary Total Hip and Knee Arthroplasty. Adv Orthop. , 9319480. Published 2019 Dec 18. doi:10.11. 